\title{
Disseminated BCG Infection in a patient with Severe Combined Immunodeficiency
}

Tae II Han, MD ${ }^{1}$

In-One Kim, MD'

Woo Sun Kim, MD²

Kyung Mo Yeon, MD²

Index words:

Bones, infection

Infants, newborn, skeletal system

Tuberculosis, gastrointestinal

Tuberculosis, musculoskeletal

Korean J Radiol 2000; $1: 114-117$

Received December 21, 1999; accepted

after revision February 24, 2000.

Department of ${ }^{1}$ Radiology, Eulji University School of Medicine; 'Radiology, Seoul National University College of Medicine,

Supported by grant 02-1995-190-0 from the Seoul National University Hospital research fund

Supported by grant 1994 from the Korean Radiological Foundation

Address reprint requests to:

In-One Kim, MD, Department of Radiology,

Seoul National University Hospital, 28,

Yongun-Dong, Chono-Gu, Seoul 110-744,

Korea.

Telephone: (822) 760-3608

Fax: (822) 747-5781
Disseminated mycobacterial infection after bacillus Calmette-Guerin (BCG) vaccination is a very rare disorder, occurring mostly in patients with immunologic deficiency. We report a case of disseminated BCG infection in a 16-month-old girl with severe combined immunodeficiency. Plain radiographs showed multiple osteolytic lesions in the femora, tibiae, humerus, and phalanges. Abdominal sonography and CT scanning revealed multiple nodules in the spleen, and portocaval lymphadenopathy.

T mmunization of children with bacillus Calmette-Guerin (BCG), a live attenuated bacterial vaccine derived from Mycobacterium bovis, is recommended by the World Health Organization in communities with a high prevalence of tuberculosis. BCG vaccines are extremely safe in immunocompetent hosts, but possible complications range from local inflammatory reactions (lymphadenitis, abscess, fistula formation) to disseminated diseases (osteomyelitis, bacteremia, meningitis) and death (1). Significant local reactions occur in $0.1-1 \%$ of vaccine recipients, whereas serious disseminated BCG infection occurs very rarely, in fewer than one in a million cases (2). The disseminated form of tuberculosis, which has a high mortality rate, develops mostly in patients with immunologic deficiency (3). Radiological reports of disseminated BCG infection have focused mainly on osteomyelitis $(3-5)$. We described the radiological manifestations of disseminated BCG infection in an infant with severe combined immunodeficiency.

\section{CASE REPORT}

A 7-month-old girl presented with a prolonged period of chronic diarrhea. On admission she showed profound abnormalities in both cell-mediated and humoral immunities and severe combined immunodeficiency was diagnosed. Laboratory values were as follows: immunoglobulin (IgG), $173 \mathrm{mg} / \mathrm{dL}$ (normal range, $500-1300 \mathrm{mg} / \mathrm{dL}$ ); IgM, $31 \mathrm{mg} / \mathrm{dl}$ (normal range, $50-199 \mathrm{mg} / \mathrm{dL}$ ); IgA, $29 \mathrm{mg} / \mathrm{dL}$ (normal range, $23-190$ $\mathrm{ml} / \mathrm{dL}$ ); T2/ T4/ T8 /B cell /Natural Killer (NK) cell, 4\% / 4\% / 0\% / 2\% / 90\%.

At the age of 10 months she was readmitted due to left axillary and cervical lymphadenopathy; several courses of antibiotic treatment had failed to change the course of her illness. There was no family history of tuberculosis, but she had been vaccinated with BCG at the age of one month. A specimen for culture was obtained from the axillary lymph node, but bacteriological culture was negative. Numerous acid-fast bacilli were demonstrated on smears, and cultures revealed growth of Mycobacterium bovis, BCG strain. She was treated with isoniazid (15 mg/kg per day), rifampicin $(15 \mathrm{mg} / \mathrm{kg}$ per day), and pyrazinamide $(20 \mathrm{mg} / \mathrm{kg}$ per day). In spite of antituberculous therapy, 

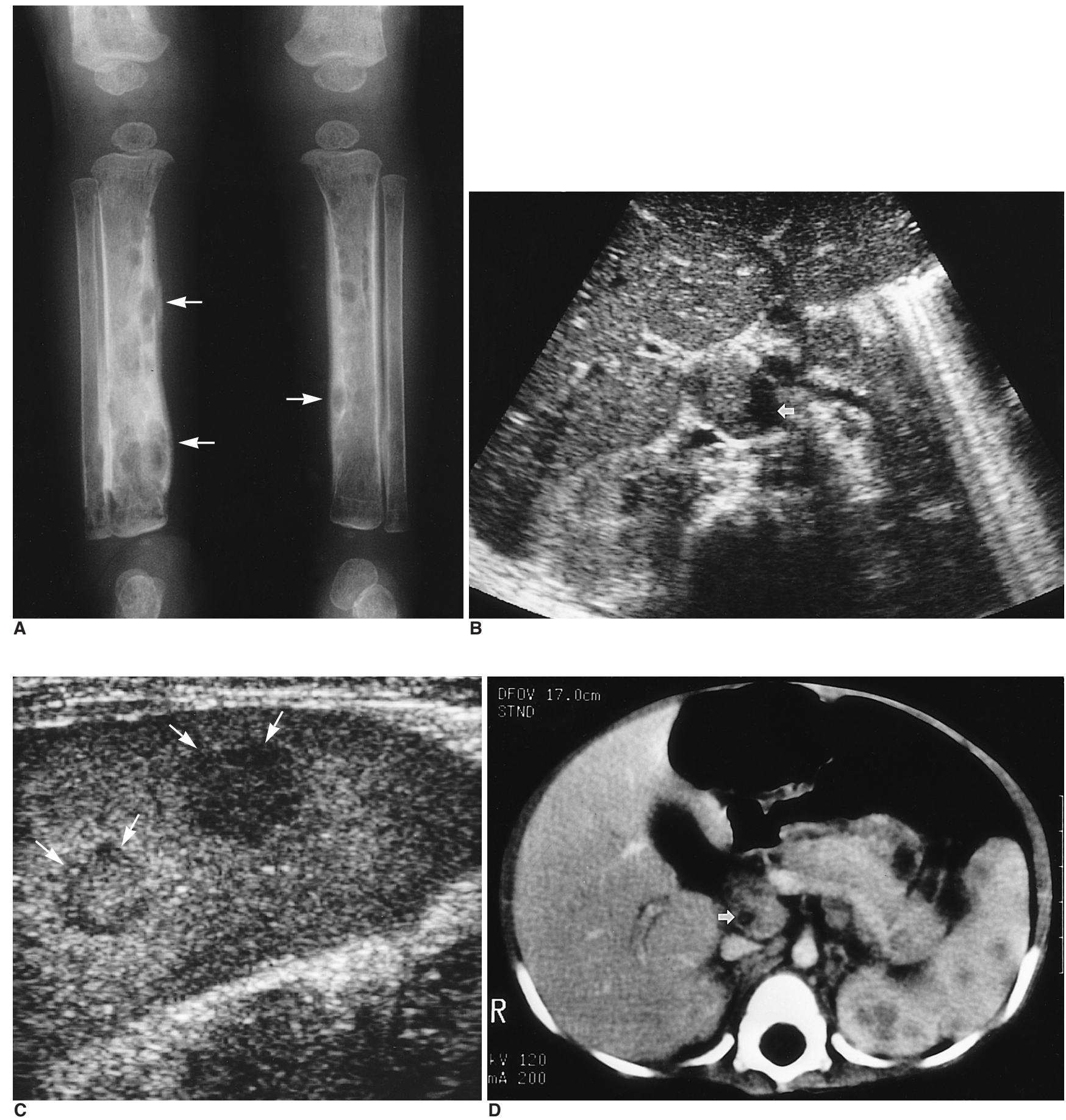

Fig. 1. Disseminated BCG infection in a 16-month-old girl with severe combined immunodeficiency.

A. Plain radiograph of both lower extremities reveals multiple, round, osteolytic lesions in the distal femora, tibiae, and fibulas. Both tibiae show extensive periosteal thickening along the entire diaphyses. Osteolytic lesions extend into the thickened periosteum (arrows).

B. Transverse sonogram through the upper abdomen shows enlarged lymph nodes containing eccentric hypoechoic portion (arrow) at the porta hepatis.

C. Longitudinal us scan of the spleen reveals hypoechoic nodules (arrows).

D. Contrast enhanced CT scan shows multiple, round, hypodense nodules in the spleen. The presence of portocaval lymphadenopathy, with a central low attenuated area (arrow), should also be noted. 
her lymphadenopathy worsened.

At the age of 16 months, she was readmitted with persistent lymphadenopathy in the left axilla and cervical area, and reddish swelling of the right third finger. A chest radiograph revealed no abnormality of lung parenchyma or hilar lymphadenopathy, but a round osteolytic lesion was incidentally detected in the right distal humerus. A plain radiograph of both lower extremities revealed multiple, round, osteolytic lesions in the femora, tibiae, and fibulas (Fig. 1A). Both tibiae showed extensive periosteal thickening along the entire diaphyses. Ultrasound examination of the abdomen showed enlarged lymph nodes containing an eccentric hypoechoic portion at the porta hepatis and hypoechoic nodules in the spleen (Figs. 1B,C). Contrast-enhanced CT scanning demonstrated the presence of multiple nodules in the spleen, and portocaval lymphadenopathy with a central low-attenuated area (Fig. 1D).

\section{DISCUSSION}

BCG is a live attenuated bacterial vaccine for infants born in countries with a high incidence of Mycobacterium tuberculosis infection, and protects children from miliary tuberculosis and tuberculous meningitis. It is considered a safe vaccine with a low incidence of complications, the overall rate of which has been estimated to be $1 \%$ or less (6). The incidence of complications, however, has varied according to the vaccine strain, dosage, and method of administration. The most frequent complications are local inflammatory reactions, including hypersensitivity, localized lymphadenitis, and local abscess or fistula formation.

Disseminated disease, the most serious complication of BCG vaccination, develops in less than one case in a million. Disseminated BCG infections have occurred following vaccination of children with immunodeficiency disorders such as severe combined immunodeficiency, chronic granulomatous disease, complete Di George syndrome, AIDS, HIV infection or idiopathic immunodeficiency of genetic origin, but only rarely in apparently normal individuals (6). Severe combined immunodeficiency, as in this case, usually presents in infancy, with severe infections, and is characterized clinically and immunologically by profound abnormalities of cell-mediated and humoral immunities. Infants with immunodeficiency contraindicate BCG vaccination, but are usually vaccinated prior to diagnosis. In other cases, immunodeficiency may be diagnosed after the development of BCG complications. Our patient was vaccinated with BCG before severe combined immunodeficiency was diagnosed. Subsequently, to prevent BCG complications, her younger brother was not vaccinated.

Disseminated BCG infection may involve the bones, joints, liver, spleen, and lymph nodes, and is usually fatal. Tuberculous osteomyelitis in infants and young children usually develops after BCG vaccination, and its frequency is assumed to be approximately one case per million vaccinations (7). Bone changes generally appear between three months and five years after vaccination. BCG osteomyelitis involves the hand, foot, long bone, spine, rib, sternum, and clavicle, the tubular bones of the fingers and toes being the most common sites of involvement (4). BCG osteomyelitis generally occurs in the metaphysis or epiphysis of the long bones or bones of the fingers and toes. In immunocompetent children, a solitary lesion with a sclerotic margin usually appears (5), whereas in children with immunodeficiency disorders there is multiple involvement. Hugosson et al. (3) reported a case of extensive disseminated skeletal osteomyelitis in a 9-month-old female infant with severe combined immunodeficiency. The bone lesions were mainly located in the metaphysis of the distal parts of the femora, proximal parts of the tibiae, both proximal humeral shafts, the ribs and skull, and no cortical thickening was observed. In our case, the involved bones were similar, except for the rib and skull, but the lesions were mainly in diaphyseal locations of the long bones, with diffuse cortical thickening.

The differential diagnoses of multiple osteolytic bone lesions in infants include metastatic neuroblastoma, leukemia, osteomyelitis, and Langerhans cell histiocytosis. Metastatic neuroblastoma and leukemia may have a similar radiographic appearance, demonstrating permeative or moth-eaten destruction and periosteal new-bone formation. Pyogenic osteomyelitis shows permeative bone destruction, and mainly involves the metaphysis. Langerhans cell histiocytosis may destroy or expand the cortex, and overlying periosteal new-bone formation is usually present, though in patients with this condition, compact periosteal reaction is also a typical feature (8).

Disseminated BCG infection in patients with severe combined immunodeficiency may also involve the lung, liver, spleen, and lymph nodes. In our case, the spleen and lymph nodes at the porta hepatis were also involved. US and CT scans of enlarged lymph nodes at the porta hepatis revealed the presence of central low echogenic or low attenuating lesions, which were thought to be areas of caseation necrosis. Demonstration of low echogenic or low attenuating areas within the enlarged lymph nodes, as demonstrated by CT scanning and ultrasound, may be helpful in the diagnosis of tuberculosis.

In summary, disseminated BCG infection in our patient with severe combined immunodeficiency led not only to disseminated osteomyelitis but also lymphadenitis and visceral organ involvement. 


\section{References}

1. Grange JM. Complications of bacille Calmette-Guerin (BCG) vaccination and immunotherapy and their management. Commun Dis Public Health 1998;1:84-88

2. Lott A. BCG complication: Estimates of the risk among vaccinated subjects and statistical analysis of their characteristics. Adv Tuberc Res 1984;21:107-193

3. Hugosson C, Harfi H. Disseminated BCG-osteomyelitis in congenital immunodeficiency. Pediatr Radiol 1991;21:384-385

4. Mortensson W, Eklof O, Jorulf H. Radiologic aspects of BCG osteomyelitis in infants and children. Acta Radiol Diagn 1976;17: 845-855

5. Arias FG, Rodriguez M, Hernandez JG, Gonzalvo P, Orense M. Osteomyelitis deriving BCG-vaccination. Pediatr Radiol 1987;17:166-167

6. Milstein JB, Gibson JJ. Quality control of BCG vaccine by WHO: a review of factors that may influence vaccine effectiveness and safety. Bull World Health Organ 1990;68:93-108

7. Nishi J, Kamenosono A, Sarker KP, Yoshino S, Ikei J, Matsuda Y. Bacille Calmette-Guerin osteomyelitis. Pediatr Infect Dis J 1997;16:332-333

8. Rogers LF. Metabolic, endocrine, and related bone diseases. In Juhl JH, Crummy AB, eds. Paul and Juhl's essentials of radiologic imaging. Philadelphia : Lippincott, 1993:262-264 